\title{
Inverse Eigenvalue Problem for a Class of Spring-Mass Systems
}

\author{
Wenting Wan*
}

School of Mathematics and Physics, Jingchu University of Technology, Jingmen, Hubei, 448000, China

\begin{abstract}
This paper discusses the constructional problem for a class of spring-mass systems whose part particles are connected to the ground. The problem is converted to an inverse eigenvalue problem for Jacobi matrix. An inverse eigenvalue problem of determining the system from its some physical parameters and incomplete eigenpairs is solved. The necessary and sufficient condition for constructing the system uniquely with positive parameters is obtained. Furthermore, the concrete expressions of the solution and the related numerical algorithm are derived, and numerical results show that the algorithm is effective.
\end{abstract}

Keywords: Eigenpair, inverse eigenvalue problem, jacobi matrix, spring-mass system.

\section{INTRODUCTION}

Problems such as the longitudinal vibration of pile are often encountered in engineering practice. Along the long direction, rod is connected to the elastic foundation in such problems, and the rod vibration system can also be dispersed into spring-mass systems by using finite difference method. Inverse eigenvalue problems for spring-mass systems are the basic problems of dynamic inverse problems in mechanics, and related research has important application in vibration control, structural design, parameters identification and so on. The inverse eigenvalue problems for spring-mass systems are using part frequency (eigenvalues) and the corresponding vibration (eigenvectors) which have been measured to determine the remaining physical parameters of systems. Such problems in mathematics are converted to inverse eigenvalue problems for Jacobi matrices. At present, research on the inverse eigenvalue problems for Jacobi matrices had some good results in [1-6]. Gladwell and Gbadeyan [7], and Nylen and Uhlig [8] considered the inverse vibration problems of spring-mass systems only from two sets of eigenvalues. Using one eigenpair or two eigenpairs, the inverse eigenvalue problems for spring-mass systems were studied by Ram and Gladwell [9], and Tian and Dai [10]. Huang, et al. [11] studied by two incomplete eigenpairs to determine a spring-mass system, and the numerical method of the problem was given. In fact, it is difficult to obtain the entire spectrum of a system from vibration test in most situations, and only some of the eigenvalues and the eigenvectors of a system are got easily. Combined with the engineering of application, this paper constructs a grounding spring-mass system that is different from [10] by some physical parameters and incomplete eigenpairs of the system. Moreover, existence condition and expressions of the solution and the corresponding numerical algorithm are obtained.
$\mathrm{N}$ degree of freedom of spring-mass system whose anterior $p$ particles are connected to the ground by springs (Fig. 1). Generalized eigenvalue equation for the system is:

$K X=\lambda M X$,

where $\lambda$ and $X$ are respectively eigenvalue and eigenvector of matrix pair $(K, M), \lambda=\omega^{2}, \omega$ is natural frequency, $X$ is vibration mode, mass matrix is:

$M=\operatorname{diag}\left(m_{1}, m_{2}, \cdots, m_{n}\right)$,

particle quality $m_{i}>0$, ungrounded spring stiffness $k_{i}>0(i=1,2, \cdots, n-1) \quad$ grounded spring stiffness $c_{j}>0(j=1,2, \cdots, p)$, stiffness matrix is:

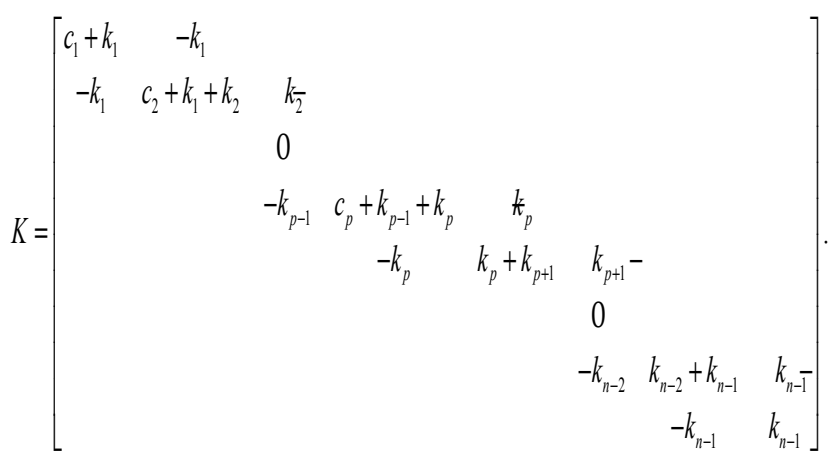

In this paper, we consider the following inverse eigenvalue problem:

Problem As for spring-mass system whose anterior $p$ particles are connected to the ground by springs, particle quality $m_{i}>0(i=p+1, p+2, \cdots, n)$ and ungrounded spring stiffness $k_{i}>0(i=1,2, \cdots, n-1)$ are known. Given

$\lambda, \mu \in \boldsymbol{R}^{+}(\lambda \neq \mu)$ and vectors 


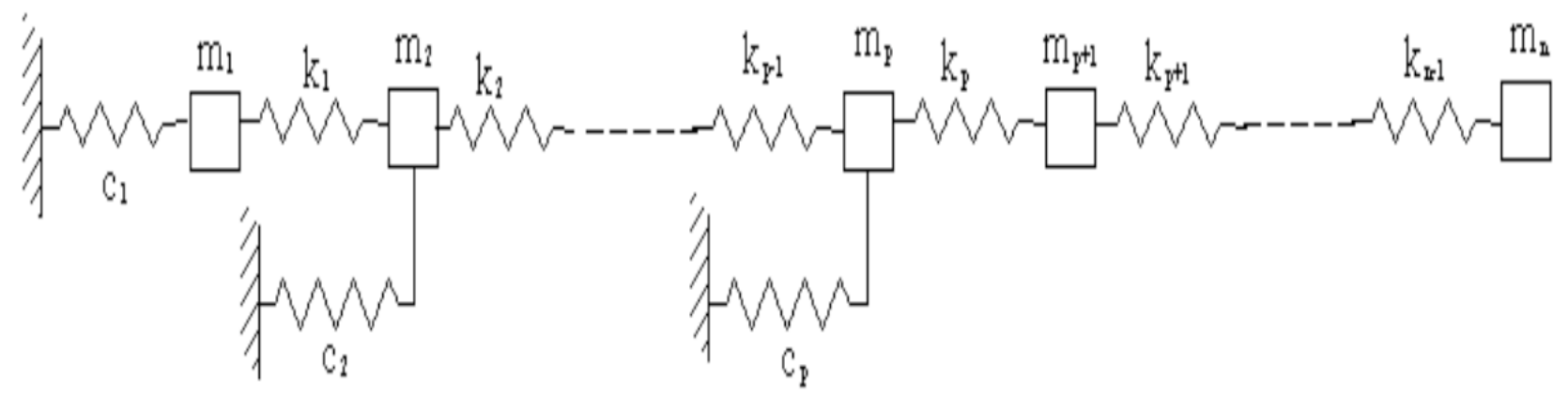

Fig. (1). A grounding spring-mass system.

$X_{1}=\left(x_{1}, x_{2}, \cdots, x_{p}\right)^{T}$,

$Y_{1}=\left(y_{1}, y_{2}, \cdots, y_{p}\right)^{T} \in \boldsymbol{R}^{p}$,

construct physical parameters of the system, that is, solve particle quality $m_{i}$, grounded spring stiffness

$c_{i}>0(i=1,2, \cdots, p)$ and vectors

$X_{2}=\left(x_{p+1}, x_{p+2}, \cdots, x_{n}\right)^{T}$,

$Y_{2}=\left(y_{p+1}, y_{p+2}, \cdots, y_{n}\right)^{T} \in \boldsymbol{R}^{n-p}$,

such that

$K X=\lambda M X, K Y=\mu M Y$,

where vectors $X$ and $Y$ are

$X=\left(X_{1}^{T}, X_{2}^{T}\right)^{T}, Y=\left(Y_{1}^{T}, Y_{2}^{T}\right)^{T} \in \boldsymbol{R}^{n}$,

$K$ and $M$ are respectively mass matrix and stiffness matrix, $(\lambda, X)$ and $(\mu, Y)$ are respectively the $\mathrm{i}^{\text {th }}$ and the $\mathrm{j}^{\text {th }}$ eigenpair of the system $\left(1 \leq i, j^{\prime} \leq n\right)$, when eigenvalues of the system are arranged in ascending order.

\section{THE ANALYSIS OF PROBLEM}

If $m_{i}>0(i=1,2, \cdots, n)$, then the equation $K X=\lambda M X$

is equivalent to the equation

$J X=\lambda X$,

where $J=M^{-1} K$ is $n \times n$ Jacobi matrix. rem.

In [12], Gladwell gave the following definition and theo-

Definition. For $n \times 1$ real vector

$X=\left(x_{1}, x_{2}, \cdots, x_{n}\right)^{T}$, sign change number of sequence which composed of the component (that value being equal to zero can be neglected ) is denoted by $S(X)$.

The eigenvalues of the Jacobi matrix are unequal real numbers, so let $\lambda_{i}>0(i=1,2, \cdots, n)$ be eigenvalues of $n \times n$ Jacobi matrix $J$.

If $\lambda_{1}<\lambda_{2}<\cdots<\lambda_{n}$, then

Lemma. Let $(\lambda, X)$ be eigenpair of $n \times n$ Jacobi matrix $J$, then $(\lambda, X)$ is the ith eigenpair of $J$ if and only if

$S(X)=i-1, i=1,2, \cdots, n$.

Matrices $K, M, X, Y$ can be expressed the following partitioned matrices.

$K=\left[\begin{array}{cc}K_{1} & -k_{p} e_{p} \varepsilon_{1}^{T} \\ -k_{p} \varepsilon_{1} e_{p}^{T} & K_{2}\end{array}\right]$,

$M=\left[\begin{array}{ll}M_{1} & \\ & M_{2}\end{array}\right]$,

$X=\left[\begin{array}{l}X_{1} \\ X_{2}\end{array}\right], Y=\left[\begin{array}{l}Y_{1} \\ Y_{2}\end{array}\right]$,

where $p \times p$ Jacobi matrix $K_{1}$ is the top left corner matrix of $K,(n-p) \times(n-p)$ Jacobi matrix $K_{2}$ is the bottom right corner matrix of $K, p \times p$ diagonal matrix $M_{1}$ is the top left corner matrix of $M,(n-p) \times(n-p)$ diagonal matrix $M_{2}$ is the bottom right corner matrix of $M, p \times 1$ unit vector

$e_{p}=(0,0, \cdots, 1)^{T}$, 
$(n-p) \times 1$ unit vector

$\varepsilon_{1}=(1,0, \cdots, 0)^{T}$,

$X_{1}, Y_{1} \in \boldsymbol{R}^{p}, X_{2}, Y_{2} \in \boldsymbol{R}^{n-p}$.

formula (1) can be rewritten as follows.

$$
\left.\begin{array}{c}
\left\{\begin{array}{c}
\lambda x_{i} m_{i}-x_{i} c_{i}=k_{i-1}\left(x_{i}-x_{i-1}\right)-k_{i}\left(x_{i+1}-x_{i}\right), \\
i=1,2, \cdots, p,
\end{array}\right. \\
\left(K_{2}-\lambda M_{2}\right) X_{2}=k_{p} x_{p} \varepsilon_{1},
\end{array}\right\}
$$

where $k_{0}=0, x_{0}=y_{0}=0$.

From (2), the problem solution can be transformed into solving the following problem I and II :

Problem I. Given $K_{2}, M_{2}$ and positive real number $\lambda$ $, \mu(\lambda \neq \mu), X_{1}, Y_{1} \in \boldsymbol{R}^{p}$ and $k_{p}$ are known, solve vector $X_{2}, Y_{2} \in \boldsymbol{R}^{n-p}$ which satisfied

$\left\{\begin{array}{l}\left(K_{2}-\lambda M_{2}\right) X_{2}=k_{p} x_{p} \varepsilon_{1}, \\ \left(K_{2}-\mu M_{2}\right) Y_{2}=k_{p} y_{p} \varepsilon_{1} .\end{array}\right.$

Problem II. Given positive real number $\lambda, \mu \quad(\lambda \neq \mu$ ) $, X_{1}, Y_{1} \in \boldsymbol{R}^{p}$ and $k_{i}(i=1,2, \cdots, p)$, solve $m_{i}$ and $c_{i}(i=1,2, \cdots, p)$, such that

$\left\{\begin{array}{c}\lambda x_{i} m_{i}-x_{i} c_{i}=k_{i-1}\left(x_{i}-x_{i-1}\right)-k_{i}\left(x_{i+1}-x_{i}\right), \\ \mu y_{i} m_{i}-y_{i} c_{i}=k_{i-1}\left(y_{i}-y_{i-1}\right)-k_{i}\left(y_{i+1}-y_{i}\right),\end{array}\right.$

$i=1,2, \cdots, p$,

where $k_{0}=0, x_{0}=y_{0}=0$.

\section{THE CONDITIONS OF UNIQUE SOLUTION OF THE PROBLEM}

Let

$\varphi_{n-p}(\gamma)=\operatorname{det}\left(K_{2}-\gamma M_{2}\right)$,

$\phi_{0}(\gamma)=1$

$\phi_{1}(\gamma)=k_{n-1}-\gamma m_{n}$ $i \times i$ determinant $\phi_{i}(\gamma)$ is the top left corner cofactor of $\phi_{n-p}(\gamma)$, that is the $i \times i$ cofactor is obtained by deleting the first $n-p-i$ columns and the first $n-p-i$ rows of determinant $\operatorname{det}\left(K_{2}-\gamma M_{2}\right)$.

Obviously, based on the result of $\varphi_{i-1}(\gamma)$, we can expand determinant $\phi_{i}(\gamma)$ by the first row and get:

$\varphi_{i}(\gamma)=\left(k_{n-i}+k_{n-i+1}-\lambda m_{n-i+1}\right) \varphi_{i-1}(\gamma)-k_{n-i+1}^{2} \varphi_{i-2}(\gamma)$,

$i=2,3, \cdots, n-p$.

Let

$z_{i}=(\lambda-\mu) x_{i} y_{i}$

$f_{i}=x_{i}\left(y_{i+1}-y_{i}\right)-y_{i}\left(x_{i+1}-x_{i}\right)$,

$g_{i}=x_{i}\left(y_{i}-y_{i-1}\right)-y_{i}\left(x_{i}-x_{i-1}\right)$,

$h_{i}=\lambda x_{i}\left(y_{i+1}-y_{i}\right)-\mu y_{i}\left(x_{i+1}-x_{i}\right)$,

$l_{i}=\lambda x_{i}\left(y_{i}-y_{i-1}\right)-\mu y_{i}\left(x_{i}-x_{i-1}\right)$,

$r_{i}=k_{i} f_{i}-k_{i-1} g_{i}$,

$s_{i}=k_{i} h_{i}-k_{i-1} l_{i}$,

$i=1,2, \cdots, p$.

Theorem 1. Systems of linear equations

$\left(K_{2}-\gamma M_{2}\right) X_{2}=k_{p} x_{p} \varepsilon_{1}$

have a unique solution if and only if

$\phi_{n-p}(\gamma) \neq 0$,

and the solution is:

$x_{i}=x_{p} \phi_{n-p}^{-1}(\gamma) \phi_{n-i}(\gamma) \prod_{j=p}^{i-1} k_{j}$,

$i=p+1, p+2, \cdots, n$.

Proof. Systems of linear equations

$\left(K_{2}-\gamma M_{2}\right) X_{2}=k_{p} x_{p} \varepsilon_{1}$

have a unique solution if and only if

$\operatorname{det}\left(K_{2}-\gamma M_{2}\right) \neq 0$,

that is

$\phi_{n-p}(\gamma) \neq 0$ 
If $\phi_{n-p}(\gamma) \neq 0$,

then,

$$
\begin{aligned}
& X_{2}=\left(x_{p+1}, x_{p+2}, \cdots, x_{n}\right)^{T} \\
& =k_{p} x_{p}\left(K_{2}-\gamma M_{2}\right)^{-1} \varepsilon_{1} \\
& =k_{p} x_{p}\left[\operatorname{det}\left(K_{2}-\gamma M_{2}\right)\right]^{-1} \cdot\left(K_{2}-\gamma M_{2}\right)^{*} \varepsilon_{1} .
\end{aligned}
$$

Because $\left(K_{2}-\gamma M_{2}\right)^{*} \varepsilon_{1}$ is algebraic cofactor of the first row elements of the matrix $K_{2}-\gamma M_{2}$,

and then from (5), we have

$$
\begin{aligned}
& X_{2}=\left(x_{p+1}, x_{p+2}, \cdots, x_{n}\right)^{T} \\
& =k_{p} x_{p} \varphi_{n-p}^{-1}(\gamma)\left[\varphi_{n-p-1}(\gamma), \varphi_{n-p-2}(\gamma) \cdot k_{p+1},\right. \\
& \varphi_{n-p-3}(\gamma) \prod_{j=p+1}^{p+2} k_{j}, \cdots, \varphi_{2}(\gamma) \prod_{j=p+1}^{n-3} k_{j}, \\
& \left.\varphi_{1}(\gamma) \prod_{j=p+1}^{n-2} k_{j}, \varphi_{0}(\gamma) \prod_{j=p+1}^{n-1} k_{j}\right]^{T} .
\end{aligned}
$$

Therefore,

$$
\begin{aligned}
& x_{i}=x_{p} \phi_{n-p}^{-1}(\gamma) \phi_{n-i}(\gamma) \prod_{j=p}^{i-1} k_{j}, \\
& i=p+1, p+2, \cdots, n .
\end{aligned}
$$

From Theorem 1, the conditions for Problem I has unique solution is easy to derive. if

Theorem 2. Problem I has a unique solution if and only

$\phi_{n-p}(\lambda) \neq 0$,

and $\phi_{n-p}(\mu) \neq 0$,

and the solution is

$$
\begin{aligned}
& x_{i}=x_{p} \varphi_{n-p}^{-1}(\lambda) \varphi_{n-i}(\lambda) \prod_{j=p}^{i-1} k_{j}, \\
& y_{i}=y_{p} \varphi_{n-p}^{-1}(\mu) \varphi_{n-i}(\mu) \prod_{j=p}^{i-1} k_{j}, \\
& i=p+1, p+2, \cdots, n .
\end{aligned}
$$$$
\text { if }
$$

Theorem 3. Problem II has a unique solution if and only $z_{i} \neq 0$,

and $z_{i}, r_{i}, s_{i}$ have the same sign, the solution is

$$
m_{i}=\frac{r_{i}}{z_{i}}, c_{i}=\frac{s_{i}}{z_{i}}
$$

$i=1,2, \cdots, p$.

Proof. Systems of linear equations (4) have a unique solution if and only if coefficient determinant

$$
\left|\begin{array}{ll}
\lambda x_{i} & -x_{i} \\
\mu y_{i} & -y_{i}
\end{array}\right| \neq 0,
$$

that is $z_{i} \neq 0$,

and the solution is

$$
\begin{aligned}
& m_{i}=\frac{r_{i}}{z_{i}}, c_{i}=\frac{s_{i}}{z_{i}}, \\
& i=1,2, \cdots, p .
\end{aligned}
$$

And for $m_{i}>0, c_{i}>0$,

$z_{i}, r_{i}, S_{i}$ have the same sign, $i=1,2, \cdots, p$.

$(\lambda, X)$ and $(\mu, Y)$ are respectively the $i^{\text {th }}$ and the $j^{\text {th }}$ eigenpair of the system $\left(1 \leq i^{\prime}, j^{\prime} \leq n\right)$, when eigenvalues of the system are arranged in ascending order. Combined with lemma, from Theorem 2 and Theorem 3, we have the necessary and sufficient condition for unique solution of the problem.

Theorem 4. Problem has a unique solution if and only if,

(1) $\phi_{n-p}(\lambda) \neq 0$ and $\phi_{n-p}(\mu) \neq 0$;

(2) $S(X)=i^{\prime}-1, S(Y)=j^{\prime}-1$;

(3) $z_{i} \neq 0$, and $z_{i}, r_{i}, s_{i}$ have the same sign, $i=1,2, \cdots, p$.

\section{NUMERICAL ALGORITHM AND EXAMPLE}

The above discussion allows us to write down the algorithm to solve Problem, presented as follows.

Algorithm. Given diverse positive real number $\lambda, \mu$

( $\lambda$ and $\mu$ are respectively the $i^{\text {th }}$ and the $\mathrm{j}^{\text {th }}$ eigenvalue of the system $\left(1 \leq i^{\prime}, j^{\prime} \leq n\right)$, when eigenvalues of the system are arranged in ascending order.),$m_{i} \in \boldsymbol{R}^{+}(i=p+1$, $p+2, \cdots, n), k_{i} \in$

$\boldsymbol{R}^{+}(i=1,2, \cdots, n-1)$, and $x_{i}, y_{i}(i=1,2, \cdots, p)$, construct $m_{i}, c_{i} \in \boldsymbol{R}^{+}(i=1,2, \cdots, p)$ and vectors

$X_{2}, Y_{2} \in \boldsymbol{R}^{n-p}:$ 
Step 1. From recurrence formula (5), compute $\phi_{i}(\lambda)$, $i=1,2, \cdots, n-p$. If $\phi_{n-p}(\lambda)=0$, go to step 7 .

Step 2. From recurrence formula (5), compute $\phi_{i}(\mu)$, $i=1,2, \cdots, n-p$. If $\phi_{n-p}(\mu)=0$, go to step 7 .

Step 3. From formula (6), compute $x_{i}$ and $y_{i}$, $i=p+1, p+2, \cdots, n$.

Step 4. Compute $S(X), S(Y)$. If $S(X) \neq i^{\prime}-1$ or $S(Y) \neq j^{\prime}-1$, go to step 7 .

Step 5. Compute $z_{i}, i=1,2, \cdots, p$. If some $z_{i}=0$, $i=1,2, \cdots, p$, go to step 7 .

Step 6. Compute $r_{i}, s_{i}, i=1,2, \cdots, p$. If $z_{i}, r_{i}, s_{i}($ $i=1,2, \cdots, p)$ have different sign, go to step 7 .

Step 7. The solution can not be determined uniquely, end the algorithm.

Step 8. From formula (7), compute $m_{i}, c_{i}$, $i=1,2, \cdots, p$,

Example. Given

$\lambda=2.2533, \mu=3.7120, n=8, p=3$,

$m_{4}=3, m_{5}=m_{6}=m_{7}=4, m_{8}=5$,

$k_{1}=1, k_{2}=k_{3}=2, k_{4}=3$,

$k_{5}=k_{6}=5, k_{7}=6$,

and $X_{1}=\left(x_{1}, x_{2}, x_{3}\right)^{T}=(-0.3336,0.5026,0.5421)^{T}$,

$Y_{1}=\left(y_{1}, y_{2}, y_{3}\right)^{T}=(0.1852,-0.8192,0.4907)^{T}$.

We need to construct $K, M$, and design a 8 degree of freedom of the grounding spring-mass system. Moreover $\lambda$ and $\mu$ are respectively the $5^{\text {th }}$ and the $7^{\text {th }}$ eigenvalue of the system.

By Algorithm, we get:

$\left\{\varphi_{i}(\lambda)\right\}_{0}^{5}=\{0.0010 e+03,-0.0053 e+03,-0.0465 e$

$+03,0.0858 e+03,1.0746 e+03,-2.6636 e+03\}$,

$\left\{\varphi_{i}(\mu)\right\}_{0}^{5}=\{0.0001 e+04,-0.0013 e+04,0.0012 e$

$+04,0.0254 e+04,-0.2049 e+04,1.0286 e+04\}$,

$\varphi_{5}(\lambda) \neq 0$ and $\varphi_{5}(\mu) \neq 0$,

$X_{2}=\left(x_{4}, x_{5}, x_{6}, x_{7}, x_{8}\right)^{T}$

$=(-0.4374,-0.1048,0.2837,0.1608,-0.1832)^{T}$,
$Y_{2}=\left(y_{4}, y_{5}, y_{6}, y_{7}, y_{8}\right)^{T}$

$=(-0.1955,0.0728,0.0176,-0.0899,0.0429)^{T}$.

The sign change number $S(X)=4, S(Y)=6$, and the results show that $\lambda$ and $\mu$ is respectively the $5^{\text {th }}$ and the $7^{\text {th }}$ eigenvalue of the system.

$\left\{z_{i}\right\}_{1}^{3}=\{0.0901,0.6006,-0.3880\}$,

$\left\{f_{i}\right\}_{1}^{3}=\{0.1802,0.6907,0.1087\}$,

$\left\{g_{i}\right\}_{1}^{3}=\{0.0000,0.1802,0.6907\}$,

$\left\{h_{i}\right\}_{1}^{3}=\{0.1802,1.6036,0.9460\}$,

$\left\{l_{i}\right\}_{1}^{3}=\{0.0901,1.4053,1.5281\}$,

$\left\{r_{i}\right\}_{1}^{3}=\{0.1802,1.2012,-1.1641\}$,

$\left\{s_{i}\right\}_{1}^{3}=\{0.1802,1.8019,-1.1644\}$,

$z_{i} \neq 0$, and $z_{i}, r_{i}, s_{i}$ have the same sign, $i=1,2,3$.

Then,

$\left\{m_{i}\right\}_{1}^{3}=\{2.0000,2.0000,3.0003\}$,

$\left\{c_{i}\right\}_{1}^{3}=\{1.9995,3.0002,3.0009\}$.

Therefore, $M=\operatorname{diag}(2,2,3.0003,3,4,4,4,5)$,

$$
K=\left[\begin{array}{ccccccccc}
2.9995 & -1 & & & & & & \\
-1 & 6.0002 & -2 & & & & & \\
& -2 & 7.0009 & -2 & & & & \\
& & -2 & 5 & -3 & & & \\
& & & -3 & 8 & -5 & & \\
& & & & -5 & 10 & -5 & \\
& & & & & -5 & 11 & -6 \\
& & & & & & -6 & 6
\end{array}\right] .
$$

Using Matlab, we get all generalized eigenvalues of $K X=\lambda M X$ are

$\sigma(K, M)=\{0.0435,0.5969,1.2430,1.5271$,

$2.2533,3.0723,3.7120,4.5018\}$.

The eigenvector which corresponds to eigenvalue $\lambda=2.2533$ is,

$X=(-0.3335,0.5026,0.5421,-0.4375$,

$-0.1048,0.2837,0.1608,-0.1832)^{T}$

and the eigenvector which corresponds to eigenvalue $\mu=3.7120$ is: 
$Y=(0.1852,-0.8193,0.4907,-0.1955$,

$0.0728,0.0176,-0.0899,0.0429)^{T}$.

These data indicate that the algorithm is very effective.

\section{CONCLUSION}

Some physical parameters of the grounding spring-mass system and two defective modes to construct real vibration system are presented in this paper. The problem comes down to the inverse eigenvalue problem for Jacobi matrix. The necessary and sufficient condition for the reconstruction of a physical vibration system with positive mass and stiffness elements from the known data is derived. And numerical algorithm and example are provided.

\section{CONFLICT OF INTEREST}

The author confirms that this article content has no conflict of interest.

\section{ACKNOWLEDGEMENTS}

This work is supported by the Key Scientific Research Project of Hubei Provincial Department of Education (No. D20134301), the Teaching Research Project of Jingchu University of Technology (No. JX2014-19)

\section{REFERENCES}

G. M. L. Gladwell, "The inverse mode problem for lumped-mass systems”, Q. J. Mech. Appl. Math., vol. 39, pp. 297-307, 1986.
H. Dai, "Inverse eigenvalue problems for Jacobian and symmetric tridiagonal matrices", Num. Math. J. Chinese Univ., vol. 12, no.1, pp. 1-13, 1990.

X] X. Y. Hu, L. Zhang, and Z. Y. Peng, "On the construction of a Jacobi matix from its defective eigen-pair and a principal submatrix”, Math. Numer. Sin., vol. 22, no. 3, pp. 345-354, 2000.

[4] Z. Y. Peng, and X. L. Han, "Constructing Jacobi matrices with prescribed ordered defective eigenpairs and a principal submatrix", J. Comput. Appl. Math., vol. 175, pp. 321-333, 2005.

[5] X. Q. Wu, and E. X. Jiang, "A new algorithm on the inverse eigenvalue problem for double dimensional Jacobi matrices", Linear Algebra Appl., vol. 437, no. 7, pp. 1760-1770, 2012.

[6] Y. Wei, "A Jacobi matrix inverse eigenvalue problem with mixed data", Linear Algebra Appl., vol. 439, no. 10, pp. 2774-2783, 2013.

[7] G. M. L. Gladwell, and J. A. Gbadeyan, "On the inverse problem of the vibrating string or rod", Q. J. Mech. Appl. Math., vol. 38, pp. 169-74, 1985.

[8] P. Nylen, and F. Uhlig, "Inverse eigenvalue problems associated with spring-mass systems", Linear Algebra Appl., vol. 254, pp. 409-425, 1997.

[9] Y. M. Ram, and G. M. L. Gladwell, "Constructing a finite element model of a vibrating rod from eigendata", J. Sound Vib., vol. 169, no. 2, pp. 229-237, 1994.

[10] X. Tian, and H. Dai, "An inverse eigenvalue problem for grounding spring-mass systems", J. Shangdong Univ. (Nat. Sci.), vol. 43, no. 1, pp. 98-102, 2008.

[11] X. T. Huang, X. Y. Hu, and L. Zhang, "On the construct design of a fixed-fixed undamped mass-spring system under the sum of mass of system restriction", Math. Appl., vol. 20, no.1, pp. 70-75, 2007.

[12] G.M.L. Gladwell, Inverse Problems in Vibration, Martinus Nijhoff Publishers: Dordrecht, the Netherland, 1986.

Received: November 28, 2014

Revised: January 10, 2015

Accepted: January 25, 2015

(C) Wenting Wan; Licensee Bentham Open.

This is an open access article licensed under the terms of the Creative Commons Attribution Non-Commercial License (http://creativecommons.org/licenses/by-nc/3.0/) which permits unrestricted, non-commercial use, distribution and reproduction in any medium, provided the work is properly cited. 